\section{Be aware of your brother}

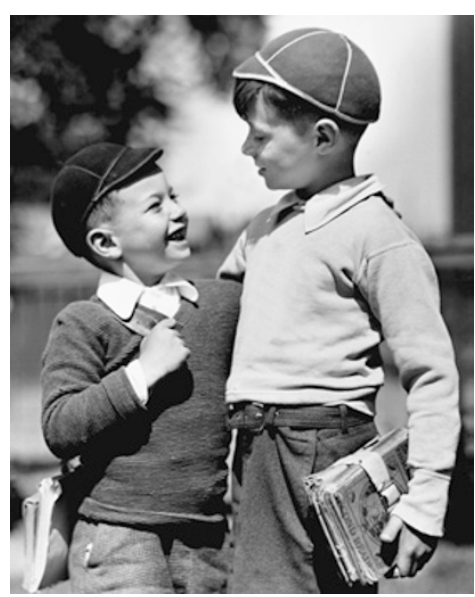

indicated that single MTL neurons might code for particular objects.

Here, the authors studied patients with epilepsy who had been implanted with electrodes as part of their evaluation for brain surgery aimed at controlling their seizures. The electrodes also enabled recording of single-neuron responses in the MTL to presentations of photos of familiar or famous people, animals and buildings. Each photo was shown multiple times and for different durations. Importantly, in contrast to previous studies, the photo presentations were so short (33 ms, $66 \mathrm{~ms}, 132 \mathrm{~ms}$ and $264 \mathrm{~ms}$ )

Consciousness is a most elusive concept, both in philosophical and in scientific terms. Some neuroscientists have tackled this by studying visual awareness and asking how activity in particular brain areas correlates with the conscious perception of a visual stimulus. Quian Quiroga et al. now show that single neurons in the medial temporal lobe (MTL) that fire in response to specific images do so only if the images are recognized, providing a neural correlate of conscious perception.

Earlier experiments by the same research group showed that single MTL neurons fire sparsely and selectively in response to 1-second-long representations of a particular face, animal, object or scene, regardless of whether the representation is realistic (for example, a photo) or abstract (for example, a written word). This that they were on the verge of conscious perception. In each trial the patients indicated whether they had perceived and recognized the image that had been presented. Using this information, the authors were able to correlate the response of individual MTL neurons to the conscious perception of particular stimuli.

The single-cell recordings confirmed the previous findings that each neuron responds to a particular picture and not (or hardly) to any other pictures. Importantly, however, a neuron only responded if the picture was recognized. For example, if a picture of a patient's brother was flashed for $33 \mathrm{~ms}$, the patient would sometimes perceive it and sometimes not. The corresponding MTL neuron fired in the trials in which the photo was perceived but not in the other trials. In other words, a single visual input triggered different responses in a specific MLT neuron, and the response depended on the patient's concious perception of the stimulus.

In most trials the neuronal response started approximately $300 \mathrm{~ms}$ after the stimulus onset, when the stimulus itself was no longer present, and lasted for up to $700 \mathrm{~ms}$; this indicates that the responses were not merely reflections of the stimulus durations.

The finding that firing of individual MTL neurons is directly related to the conscious perception of a specific image extends an earlier report of single-neuron responses to visual-stimulus perception in humans. The long latency between stimulus presentation and neuronal response indicates that MTL neuron firing probably does not underlie the actual perception of the stimulus: taking into account the role of the MTL in memory, the authors suggest that MTL neurons might have a role in storing consciously perceived visual inputs in long-term memory.

Leonie Welberg

ORIGINAL RESEARCH PAPER Quian Quiroga, R. et al. Human single-neuron responses at the threshold of conscious recognition. Proc. Natl Acad. Sci. USA 105, 3599-3604 (2008) FURTHER READING Quian Quiroga, R. et al. Invariant visual representation by single neurons in the human brain. Nature 435, 1102-1107 (2005)| Kreiman, G. et al. Single-neuron

correlates of subjective vision in the human medial temporal lobe. Proc. Natl Acad. Sci. USA 99, 8378-8383 (2002) 